\title{
QuASI-INTEGRALS OF THE Plane Restricted THREE-BODY Problem
}

\author{
E.M. Nezhinskij \\ Institute of Theoretical Astronomy of the Russian Academy \\ of Sciences, St. Petersburg
}

\begin{abstract}
A BSTRACT
The paper is concerned with studying the domain of possible motion and a field of the test body velocities in the plane restricted problem of three bodies. The study is based on existence of a quasi-integral of areas (similar to an integral of areas in $t$ he problem of $t$ wo bodies) as well as on the Jacobi integral. The method of constructing the quasiintegrals is a standard one (see, for example, [1],[2].
\end{abstract}

\section{QJA SI-I NTEGRALS}

\subsection{Prel iminary material}

Let us consider a plane dynamic system, consisting of the $t$ wo point bodies of non-zero masses and a test body. We assume that point bodies move around their barycenter in circular orbits and a test body is moving around one of these bodies.

We denote by $M_{1}$ and $M_{2}$ masses of the point bodies, by $R$ the distance between the point bodies, by 1 distance between the point body of the mass $M_{1}$ and barycenter, by $H$ distance between the point body of the mass $M_{1}$ and the libration point $L_{1}$, by $n$ the angular velocity of the point bodies, by $\mu$ ratio $M_{1} /\left(M_{1}+M_{2}\right)$. Assume, for definiteness, that a point body is moving around a body of the mass $M_{1}$. Further, we introduce the plane coordinate system $x y$ (see $F i g .1$ ) rotating with a constant angular velocity $n$, having origin in $M_{1}$, axis $x$, crossing $M_{2}$ and such unit of distance that for a test body $\max \left[x^{2}(t)+y^{2}(t)\right]=1$. 


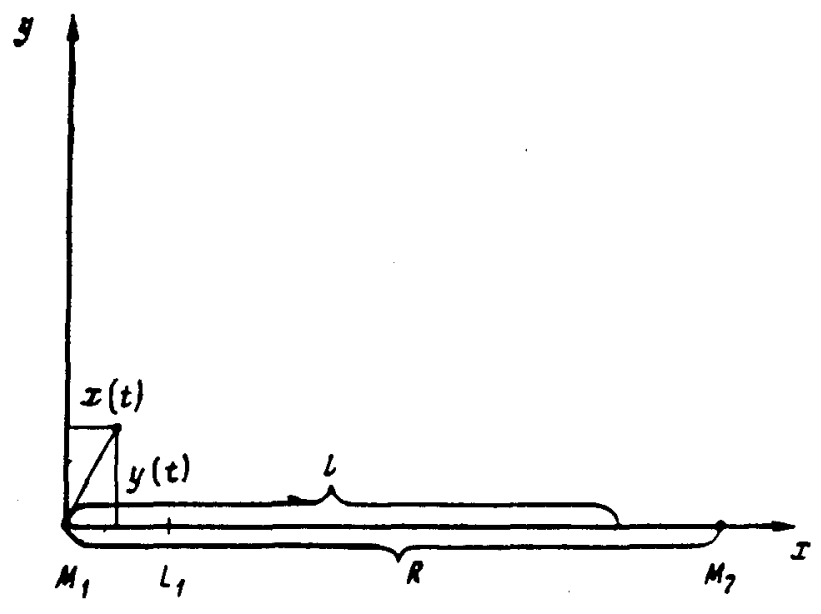

Fig. 1: Coordinate System

It may be easily proved that equations of motion for a test body in such restricted circular problem of three bodies under appropriate choice of the time unit have the form:

$$
\begin{aligned}
& \ddot{x}-2 n \dot{y}-n^{2}(x-1)=\frac{\partial}{\partial x}\left[\frac{\mu}{\sqrt{x^{2}+y^{2}}}+\frac{1-\mu}{\sqrt{(R-x)^{2}+y^{2}}}\right], \\
& \ddot{y}+2 n \dot{x}-n^{2} y=\frac{\partial}{\partial y}\left[\frac{\mu}{\sqrt{x^{2}+y^{2}}}+\frac{1-\mu}{\sqrt{(R-x)^{2}+y^{2}}}\right],
\end{aligned}
$$

where

$$
\left\{\begin{array}{l}
n=R^{-3 / 2} \\
1=R(1-\mu)
\end{array}\right.
$$

together with Bolzmann's equation, whose particular solutions are integrals of the system of equations (1) have the form:

$$
\frac{\partial f}{\partial x} \dot{x}+\frac{\partial f}{\partial y} \dot{y}+\frac{\partial f}{\partial \dot{x}}\left\{\frac{2}{R^{3 / 2}} \dot{y}+\frac{x}{R^{3}}+\frac{1-\mu}{R^{2}}-\frac{\mu x}{\left(x^{2}+y^{2}\right)^{3 / 2}}\right.
$$




$$
\begin{gathered}
+\frac{(1-\mu)(R-x)}{\left((R-x)^{2}+y^{2}\right)^{3 / 2}} \\
+\frac{\partial f}{\partial \dot{y}}\left\{\frac{2}{R^{3 / 2}} \dot{x}+\frac{y}{R^{3}}-\frac{\mu y}{\left(x^{2}+y^{2}\right)^{3 / 2}}-\frac{(1-\mu) y}{\left((R-x)^{2}+y^{2}\right)^{3 / 2}}\right\}=0
\end{gathered}
$$

It is known that for the system of equations (1) there exists Jacobi integral. In addition to this classical integral we shall attempt constructing approximate integrals i.e. quasi-integrals for (1). lities

In this paper we assume that one accomplishes the inequa-

$$
\left\{\begin{array}{l}
1<\mathrm{H}, \\
1<\mathrm{R}
\end{array}\right.
$$

We introduce a smali parameter $\gamma=\sqrt{1 / \bar{R}}$; decompose the coefficients by $\frac{\partial f}{\partial \dot{x}}$ and $\frac{\partial f}{\partial \dot{y}}$ from (2) into series in powers of $\gamma$; then the euqation (2) may be rewritten in the form

$$
\begin{aligned}
\frac{\partial f}{\partial x} \dot{x} & +\frac{\partial f}{\partial y} \dot{y}+\frac{\partial f}{\partial \dot{x}}\left\{-\frac{\mu x}{\left(x^{2}+y^{2}\right)^{3 / 2}}+\gamma^{3} 2 \dot{y}+\gamma^{6}(3-2 \mu) x+\ldots\right\}+ \\
& +\frac{\partial f}{\partial \dot{y}}\left\{-\frac{\mu y}{\left(x^{2}+y^{2}\right)^{3 / 2}} \gamma^{3} 2 x+\gamma^{6} \mu y+\ldots\right\}=0
\end{aligned}
$$

Let a single-valued particular solution of the equation (3), having the form

$$
\sum_{i=0}^{\infty} f_{i}(x, y, \dot{x}, \dot{y}) \gamma^{i}=\text { const. },
$$

be called a quasi-integral of the order q of a test body of the circular restricted three-body problem.

1.2 Jacobi's integral and quasi-integral.

A simple verification shows that the relation

$$
\begin{aligned}
0.5\left(\dot{x}^{2}+y^{2}\right)-0.5\left(x^{2}+y^{2}\right) n^{2} & +n^{2} 1 x-\frac{\mu}{\left(x^{2}+y^{2}\right)^{1 / 2}}- \\
& -\frac{1-\mu}{\left((R-x)^{2}+y^{2}\right)^{1 / 2}}=h
\end{aligned}
$$


where $h=$ const. is an integral. It is the Jacobi integral of restricted circular problem of three bodies. Let us rewrite (5) leaving the terms to the sixth order inclusive:

$0.5\left(\dot{x}^{2}+\dot{y}^{2}\right)-\frac{\mu}{\left(x^{2}+y^{2}\right)^{1 / 2}}-\gamma^{2}(1-\mu)-\gamma^{6}\left((1.5-\mu) x^{2}+\frac{\mu y^{2}}{2}\right)=h$

It is easy to see that (6) is a quasi-integral of the order 6 . Let us name this quasi-integral as the Jacobi quasi-integral.

1.3 Quasi-integral of the areas.

The equation

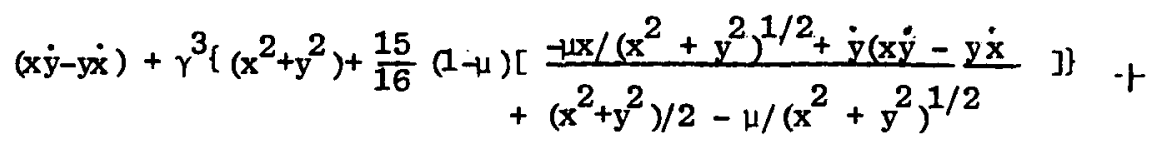

$\gamma^{6}\left\{3(1-\mu) \int x y d t+15 \frac{1-\mu}{4}\right.$

$\left.x \int \frac{\left(y \dot{y}^{2}-y \dot{x}^{2}+2 x \dot{x} \dot{y}\right)\left(-\mu x /\left(x_{i}^{2}+y^{2}\right)^{1 / 2}+\dot{y}(x \dot{y}-y \dot{y})\right.}{\left(\left(\dot{x}^{2}+\dot{y}^{2}\right) / 2-\mu /\left(x^{2}+y^{2}\right)^{1 / 2}\right)^{2}} d t\right\}=C$

where $C=$ const. shows that it is a quasi-integral of the or der 6. Te call it quasi-integral of the areas of a test body of the restricted circular problem of three-bodies (if $\gamma \Rightarrow 0$, then (7) becomes the integral of areas $\mathbf{x} \dot{y}-\dot{y} \dot{x}=C$ ).

Procedures of constructing the quasi-integral (7) consists in a direct substitution of (4) into (3) and by selec$t$ ing the terms at equal powers $\gamma$ and searching the singlevalued particular solutions of the equations obtained. Thus, function $f_{o}$ should be a particular solution of the equation.

$$
\frac{\partial f_{o}}{\partial x} \dot{x}+\frac{\partial f_{o}}{\partial y} \dot{y}-\frac{\partial f_{o}}{\partial \dot{x}} \frac{\mu x}{\left(x^{2}+y^{2}\right)^{3 / 2}}-\frac{\partial f_{o}}{\partial \dot{y}} \frac{\mu y}{\left(x^{2}+y^{2}\right)^{3 / 2}}=0 .
$$

Obviously

$$
f_{0}=x \dot{y}-\dot{y x}
$$

is the particular solution of (8). Similarly, one finds

$$
f_{1} \equiv 0, \quad f_{2} \equiv 0
$$

For function $f_{3}$ one obtains, by taking into account (9) and (10), the equation 


$$
\begin{aligned}
\frac{\partial f_{3}}{\partial x} \dot{x}+\frac{\partial f_{3}}{\partial y} \dot{y}-\frac{\partial f_{3}}{\partial \dot{x}} \frac{\mu x}{\left(x^{2}+y^{2}\right)^{3 / 2}} & -\frac{\partial f_{3}}{\partial \dot{y}} \frac{\mu y}{\left(x^{2}+y^{2}\right)^{3 / 2}} \\
& -2(x \dot{x}+y \dot{y})=0
\end{aligned}
$$

for its particular solution

$$
f_{3}=x^{2}+y^{2}
$$

Similarly as for functions $f_{1}$ and $f_{2}$ one $f 1$ nds

$$
\mathbf{f}_{4} \equiv 0, \quad \mathbf{f}_{5} \equiv 0 \text {. }
$$

For $f_{6}$ the equations (if one considers (9), (10), (12) and (13) $)^{6}$ has the form

$$
\begin{gathered}
\frac{\partial f_{6}}{\partial x} \dot{x}+\frac{\partial f_{6}}{\partial y} \dot{y}-\frac{\partial f_{6}}{\partial \dot{x}} \frac{\mu x}{\left(x^{2}+y^{2}\right)^{3 / 2}}-\frac{\partial f_{6}}{\partial \dot{y}} \frac{\mu y}{\left(x^{2}+y^{2}\right)^{3 / 2}} \\
-3(1-\mu) x y=0 . \\
f_{6}=3(1-\mu) \int x y d t
\end{gathered}
$$

is (see Supplementary notes) the solution of (14). However, if in (15) one passes to osculating elements (see, for example, [3]) then after integrating we obtain

$$
\begin{aligned}
f_{6}= & 3(1-\mu)\left(a^{7} / m\right)^{1 / 2}\left(\left(1-e^{2}\right)^{1 / 2}\left(\cos ^{2} \omega-\sin ^{2} \omega\right)[\text { e cos } E\right. \\
& \left.+\frac{1}{3} e \cos ^{3} E-\frac{1}{2}\left(1-e^{2}\right) \cos ^{2} E\right]+\cos \omega \sin \omega\left[\frac{5}{2} e^{2} E\right. \\
& \left.\left.-e\left(3+e^{2}\right) \sin E+\frac{1}{3} e\left(2-e^{2}\right) \sin ^{3} E+\frac{1}{4}\left(2+e^{2}\right) \sin 2 E\right]\right\}
\end{aligned}
$$

Since

$$
E=(-1)^{k} \arcsin \frac{x \dot{x}+y \dot{y}}{\left[\frac{\mu^{2}}{2 \mu /\left(x^{2}+y^{2}\right)^{1 / 2}-\left(\dot{x}^{2}+\dot{y}^{2}\right)}-(x \dot{y}-y \dot{x})\right]^{1 / 2}}+k \pi
$$

(where $k=0, \pm 1, \pm 2, \ldots$ ), - the function thus obtained $f_{6}$ is ambiguous. (In it one has a summand the term) 


$$
\left.\frac{15}{2}(1-\mu)\left(a^{7} / \mu\right)^{1 / 2} \cos \omega \sin \omega e^{2} E\right) \text {. }
$$

In order to find the desired quasi-integral of areas of order 6 , instead of (12) one chooses the following single-valued particular solution of equation (11) (in substance, one introduce restrictions on some kinds of symmetry):

$f_{3}=x^{2}+y^{2}+\frac{15}{16}(1-\mu)\left[\frac{\frac{\mu x}{\left(x^{2}+y^{2}\right)^{1 / 2}}+\dot{y}(x \dot{y}-y \dot{x})}{0.5\left(\dot{x}^{2}+\dot{y}^{2}\right)+\mu /\left(x^{2}+y^{2}\right)^{1 / 2}}\right]^{2}$

and instead of (15)

$$
\begin{gathered}
f_{6}=3(1-\mu) \int x y d t+\frac{15}{4}(1-\mu) \int\left(y \dot{y}^{2}-y \dot{x}^{2}+2 x \dot{x} \dot{y}\right) \\
\quad \times \frac{-\frac{\mu x}{\left(x^{2}+y^{2}\right)^{1 / 2}}+\dot{y}(x \dot{y}-y \dot{x})}{\left(0.5\left(\dot{x}^{2}+\dot{y}^{2}\right)-\mu /\left(x^{2}+y^{2}\right)^{1 / 2}\right)^{2}} d t .
\end{gathered}
$$

\subsection{Addition}

By the method presented in section 1.3 , one can show that there is no quasi-integral in the system (1), which at $\gamma \Rightarrow 0$ is turned into Laplace's integral.

\section{THE VELOC ITY FIELD}

Theorem 1. Let $C$ and $h$ be the constants of the quasiintegral of areas and of the Jacobi-quasi-integral of a test body in the restricted circular three-body problem. If $C \neq 0$, $h<0$ and $\mu^{2}+2 h C^{2} \geq 0$, then inthe vicinity of the mass $M_{1}$ the velocity field is doūble-valued.

Proof. First assume that $\dot{x}, \dot{y}$ do not depend on $\gamma$, i.e.

$$
\begin{aligned}
& \dot{x}=A_{0}(x, y), \\
& \dot{y}=B_{0}(x, y) .
\end{aligned}
$$

Then, it follows from (6) and (7), that possible are two pairs of functions: 


$$
\begin{aligned}
& A_{0}=-\frac{C y}{x^{2}+y^{2}} \pm \frac{x \operatorname{sign} x}{x^{2}+y^{2}}\left[-c^{2}+2\left(h+\frac{\mu}{\left(x^{2}+y^{2}\right)^{1 / 2}}\right)\left(x^{2}+y^{2}\right)\right]^{1 / 2} \\
& B_{0}=-\frac{C x}{x^{2}+y^{2}} \pm \frac{y \operatorname{sign} x}{x^{2}+y^{2}}\left[-c^{2}+2\left(h+\frac{\mu}{\left(x^{2}+y^{2}\right)^{1 / 2}}\left(x^{2}+y^{2}\right)\right]^{1 / 2}\right.
\end{aligned}
$$

Introduce the following parameters

$$
\begin{aligned}
& r=\left(x^{2}+y^{2}\right)^{1 / 2} \\
& \Delta=\left(-c^{2}+2(h+/ r) r^{2}\right)^{1 / 2}
\end{aligned}
$$

Now we assume that $\dot{x}, \dot{y}$ depends upon $\gamma$ and upto order of $\gamma^{4}$, we take

$$
\begin{aligned}
\dot{x}= & -C y / r^{2}+A_{1} \gamma+A_{2} \gamma^{2}+A_{3} \gamma^{3}+A_{4} \gamma^{4} \\
& \pm \frac{x \operatorname{sign} x}{r^{2}}\left[\Delta^{2}+E_{1} \gamma+B_{2} \gamma^{2}+B_{3} \gamma^{3}+B_{4} \gamma^{4}\right]^{1 / 2} \\
\dot{y}= & -C x / r^{2}+E_{1} \gamma+E_{2} \gamma^{2}+E_{3} \gamma^{3}+E_{4} \gamma^{4}+ \\
& \pm \frac{y \operatorname{sign} x}{r^{2}}\left[\Delta^{2}+F_{1} \gamma+F_{2} \gamma^{2}+F_{3} \gamma^{3}+F_{4} \gamma^{4}\right]^{1 / 2}
\end{aligned}
$$

Substituting (21) into (6) and (7) and successively retaining terms for $\gamma, \gamma^{2}, \gamma^{3}$ and $\gamma^{4}$, we find the functions

$$
\begin{aligned}
& A_{1}=B=E_{1}=F_{1}=0 ; \\
& A_{2}=E_{2}=0 \\
& E_{2}=F_{2}=2(1-\mu) r^{2} ; \\
& A_{3}=W_{2} C x^{2} y / 2 r^{4} \Delta^{2}+W_{1} y / r^{2}, \\
& E_{3}=W_{2} C y^{2} x / 2 r^{4} \Delta^{2}+W_{1} x / r^{2}, \\
& B_{3}=W_{2} y^{2} / r^{2}+W_{1} 2 C, \\
& F_{3}=W_{2} x^{2} / r^{2}+W_{1} 2 C,
\end{aligned}
$$

where 
$W_{1}=r^{2}+\frac{15}{16} \frac{1-\mu}{h^{2}}\left[\left\{-\frac{\mu x}{r}+\frac{c^{2} x}{r^{2}}\right\}^{2}+\frac{c^{2} y^{2} \Delta^{2}}{r^{4}}\right]$

$W_{2}=\frac{15}{4} \frac{1-\mu}{h^{2}} \Delta^{2} C\left[-\frac{\mu}{r}+\frac{C^{2}}{r^{2}}\right] ;$

$A_{4}=B_{4}=E_{4}=F_{4}=0$.

DOMAIN OF MOTION OF A TEST BODY

Denote by $S_{1}$ the domain of a possible motion of a test body in the vicinity of the point of mass $M_{1}$ while computing the boundaries of this domain we have taken into account the terms containing $\gamma^{1}$.

Theorem 2: Let $C$ and $h$ be constants of the quasi-integral of areas and those of the Jacobi quasi-integral in the restricted circular three-body problem. If $\mathrm{C} \neq 0$ and $\mathrm{h}<0$,

with $\mu^{2}+2 h C^{2}=0$ the domain $S_{3}$ is a circular ring

$\frac{-\mu+}{2\left(h+1(1-\mu) \gamma^{2}+C \gamma^{2}\right)} \leq r \leq \frac{-\mu-\left(2 C^{2} \gamma^{2}(1-\mu+C \gamma)^{1 / 2}\right.}{2\left(h+(1-\mu) \gamma^{2}+C \gamma^{3}\right)}$

with $\mu^{2}+2 h C^{2}>0$ the domain $S_{3}$ is an elliptical ring (indices $i$ and $j$ are used to denote $e^{3}$ iner and outer boundaries of the ring) having main axes

$$
\begin{aligned}
& a_{i(e)}=r_{2 i(e)}-r_{i(e)}^{2} \frac{c}{2 h r_{i(e)}+\mu}\left[1+\frac{15}{16}(1-\mu)\right. \\
& \times \frac{\left(\mu r_{i(e)}-c^{2}\right)^{2}}{h^{2} r_{i(e)}^{4}} j \gamma^{3} \\
& b_{i(e)}=r_{2 i(e)}^{2}-\frac{c}{2 h r_{i(e)}+\mu} r^{3} \text {, }
\end{aligned}
$$

where

$$
\begin{aligned}
& r_{i(e)}=\left(-\mu( \pm)\left(\mu^{2}+2 h C^{2}\right) / 2 h,\right. \\
& r_{2 i(e)}=\left(1+\frac{1-\mu}{h} \gamma^{2}\right) \frac{-\mu( \pm)}{2 h} \\
& \text { (t) } \frac{\left(\mu^{2}+2 h C^{2}\right)^{1 / 2}}{2 h\left(\mu^{2}+2 h C^{2}\right)^{1 / 2}} \gamma^{2}
\end{aligned}
$$


The first part of the theorem follows from the formulae (20)-(25). Let us prove the second part of the theorem.

It follows from the formulae (20)-(22) that the boundary $S_{1}$ can be computed by formula (28); from formulae (20)-(23) it follows that the boundary $S_{2}$ can be computed by formula (29). Then, it follows from formulae (20)-(24) that the boundary $S_{3}$, which is described by the end of vector $r_{3 i(e)}$ with the origin in $M_{1}$ and with the length

$$
r_{3 i(e)}\left(x_{i(e)} y_{i(e)}\right)=r_{i(e)}+\rho_{i(e)}\left(x_{i(e)}, y_{i(e)}\right) r^{3}
$$

is followed by the equality

$$
\left(2 h_{i(e)}+\mu\right) \rho_{i(e)}+c\left[r_{i(e)}^{2}+\frac{15}{16} \cdot(1-\mu) \frac{\left(\mu r_{i(e)}-c^{2}\right) x_{i(e)}^{2}}{\left.h^{2} r_{i(e)}^{4}\right]=0}\right.
$$

Equation ( 30$)$ by virtue of (31) and (20) may be rewritten in the form

$$
\begin{aligned}
r_{3 i(e)}=r_{2 i(e)} & -\left\{\frac { x _ { i ( e ) } ^ { 2 } } { 2 h _ { r _ { j ( e ) } + \mu } } C \left[1+\frac{15}{i 6}(1-\mu) \frac{\left(\mu r_{i(e)}-c^{2}\right)^{2}}{\left.h^{2} r_{i(e)}^{4}\right]}\right.\right. \\
& \left.+\frac{y_{i(e)}^{2} c}{2 h r_{i(e)}+\mu}\right\} r^{3} .
\end{aligned}
$$

It remains to note that the end of the radius-vector $r_{3 i(e)}$
sets an ellipse with main axes (27).

Consequence 1 . $S_{1}$ and $s_{2}$ are circular rings.

Consequence 2. $s_{2} \subset s_{3} I_{C>0}, s_{2}>s_{3} b_{c}, s_{3} b<0<s_{3} b>0$ (see Fig. 2)

The first consequence follows from (28) and (29); the second one - from (27) and the obvious inequalities

$$
\begin{aligned}
& 2 \mathrm{hr}_{\mathrm{e}}+\mu<0, \\
& 2 \mathrm{hr}_{1}+\mu>0 .
\end{aligned}
$$

SUPPLEMENTARY NOTES

Let us pass in (14) from $x, y, \dot{x}, \dot{y}$ to the osculating el ements a,e,w, M i.e. 


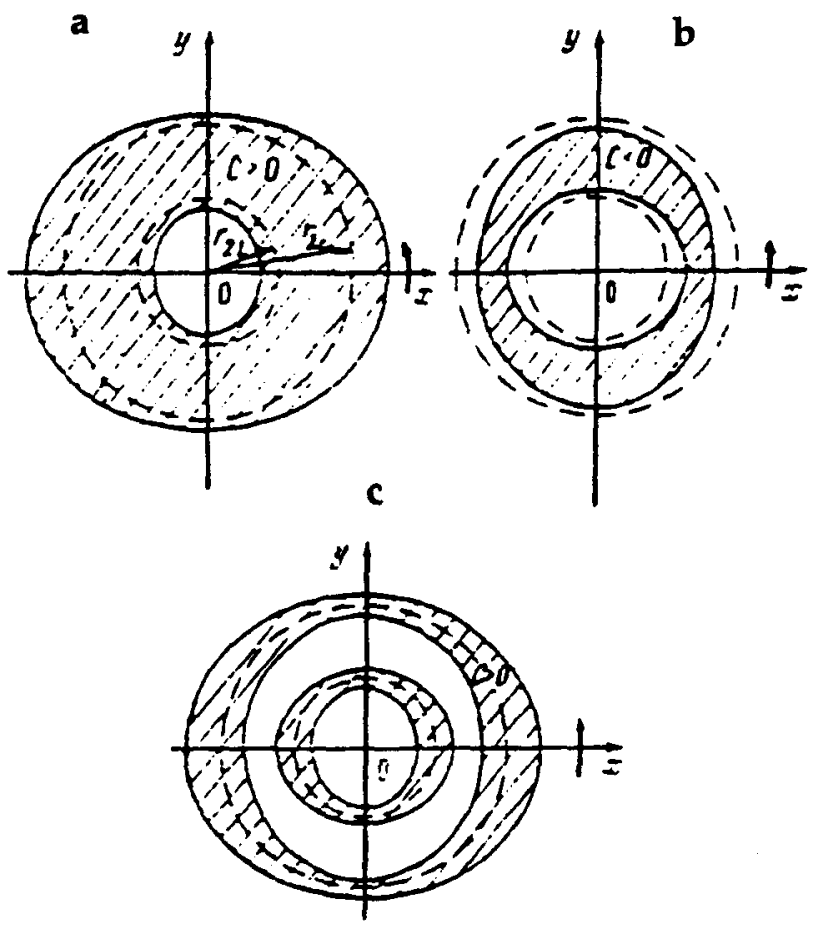

Fig. 2: The domain $\left.S_{3}\right|_{C>0}(a) ;\left.s_{3}\right|_{C<0}(b)$, and $\left.\mathrm{s}_{3}\right|_{\mathrm{C}>0} /\left.\mathrm{s}_{3}\right|_{\mathrm{C}<0}$ (c) if $|\mathrm{C}|=$ const., $\mathrm{h}=$ const.

$$
\begin{aligned}
& \frac{\partial f_{6}}{\partial x} \dot{x}+\frac{\partial f_{6}}{\partial y} \dot{y}-\frac{\partial f_{6}}{\partial \dot{x}} \frac{\mu x}{\left(x^{2}+y^{2}\right)^{3 / 2}}-\frac{\partial f_{6}}{\partial \dot{y}} \frac{\mu y}{\left(x^{2}+y^{2}\right)^{3 / 2}} \\
& =\frac{\partial f_{6}}{\partial a}\left[\frac{\partial a}{\partial x} \dot{x}+\frac{\partial a}{\partial y} \dot{y}-\frac{\partial a}{\partial \dot{x}}-\frac{\partial a}{\partial \dot{x}} \frac{\mu x}{\left(x^{2}+y^{2}\right)^{3 / 2}}-\frac{\partial a}{\partial \dot{y}} \frac{\mu y}{\left(x^{2}+y^{2}\right)^{3 / 2}}\right] \\
& \quad+\frac{\partial f_{6}}{\partial e}\left[\frac{\partial e}{\partial x} \dot{x}+\frac{\partial e}{\partial y} \dot{y}-\frac{\partial e}{\partial \dot{x}} \frac{\mu x}{\left(x^{2}+y^{2}\right)^{3 / 2}}-\frac{\partial e}{\partial \dot{y}} \frac{\mu y}{\left(x^{2}+y^{2}\right)^{3 / 2}}\right] \\
& +\frac{\partial f_{6}}{\partial \omega}\left[\frac{\partial \omega}{\partial x} \dot{x}+\frac{\partial \omega}{\partial y} \dot{y}-\frac{\partial \omega}{\partial \dot{x}} \frac{\mu x}{\left(x^{2}+y^{2}\right)^{3 / 2}}-\frac{\partial \omega}{\partial \dot{y}} \frac{\mu y}{\left(x^{2}+y^{2}\right)^{3 / 2}}\right] \\
& \quad+\frac{\partial f_{6}}{\partial M}\left[\frac{\partial M}{\partial x} \dot{x}+\frac{\partial M}{\partial y} \dot{y}-\frac{\partial M}{\partial \dot{x}} \frac{\mu x}{\left(x^{2}+y^{2}\right)^{3 / 2}}-\frac{\partial M}{\partial \dot{y}} \frac{\mu y}{\left(x^{2}+y^{2}\right)^{3 / 2}}\right]
\end{aligned}
$$


By using well known formulae

$$
\begin{aligned}
& \left(\mu a(1-e)^{2}\right)^{1 / 2}=x \dot{y}-\dot{y x} \\
& \frac{2 \mu}{a(1-e \cos E)}-\frac{\mu}{a}=\dot{x}^{2}+\dot{y}^{2}, \\
& a(\cos E-e)=x \cos \omega+y \sin \omega \text {, } \\
& a\left(1-e^{2}\right) \sin E=-x \sin \omega+y \cos \omega \text {, } \\
& \mathrm{H}=\mathrm{E}-\mathrm{e} \cos \mathrm{E} \text {, }
\end{aligned}
$$

one can show that in (32).

$$
\begin{aligned}
& \frac{\partial a}{\partial x} \dot{x}+\frac{\partial a}{\partial y} \dot{y}-\frac{\partial a}{\partial \dot{x}} \frac{\mu x}{\left(x^{2}+y^{2}\right)^{3 / 2}}-\frac{\partial a}{\partial \dot{y}} \frac{\mu y}{\left(x^{2}+y^{2}\right)^{3 / 2}}=0, \\
& \frac{\partial e}{\partial x} \dot{x}+\frac{\partial e}{\partial y} \dot{y}-\frac{\partial e}{\partial \dot{x}} \frac{\mu x}{\left(x^{2}+y^{2}\right)^{3 / 2}}-\frac{\partial e}{\partial \dot{y}} \frac{\mu y}{\left(x^{2}+y^{2}\right)^{3 / 2}}=0, \\
& \frac{\partial \omega}{\partial x} \dot{x}+\frac{\partial \omega}{\partial y} \dot{y}-\frac{\partial \omega}{\partial \dot{x}} \frac{\mu x}{\left(x^{2}+y^{2}\right)^{3 / 2}}-\frac{\partial \omega}{\partial \dot{y}} \frac{\mu y}{\left(x^{2}+y^{2}\right)^{3 / 2}}=0, \\
& \frac{\partial M}{\partial x} \dot{x}+\frac{\partial M}{\partial y} \dot{y}-\frac{\partial M}{\partial \dot{x}} \frac{\mu x}{\left(x^{2}+y^{2}\right)^{3 / 2}}-\frac{\partial M}{\partial \dot{y}} \frac{\mu y}{\left(x^{2}+y^{2}\right)^{3 / 2}}=0
\end{aligned}
$$

\section{REF ER ENCES}

[1] Timoshkova; E.I., Kholchevnikov, K.V. //Itogi nauki i tekhniki. Ser. Astronomia, 1982, T.20, p.19.

[2] Contopoulos $G ., / /$ The Astrophys. J. 1965, V.142,p.8 02 .

[3] Subbotin,M.F. Vvedenie $v$ teoret icheskuyu a stronomi yu. Moscow, Nauka, 1968. 